\title{
Incidentes críticos de la participación en el aula: explorando la voz de estudiantes del programa de integración escolar
}

\author{
Critical Incidents in the classroom: Exploring student voices in \\ school integration programs
}

Incidentes críticos da participação na sala de aula: explorando a voz dos estudantes do programa de integração escolar

\author{
Ignacio Figueroa-Céspedes* , Nataly Arévalo-Aróstica** \\ Cristopher Yáñez-Urbina ${ }^{* * *}$, Fernanda Muñoz-Abarca ${ }^{* * * *}$
}

\begin{abstract}
RESUMEN
El presente artículo describe y analiza los incidentes críticos reportados por 24 estudiantes de séptimo básico a tercero medio, pertenecientes a programas de integración de escuelas en situación de vulnerabilidad de Santiago y La Serena, Chile. Es una investigación cualitativa de diseño narrativo y de alcance descriptivo. La información se recogió a través de pautas de incidentes críticos, dibujos y entrevistas semiestructuradas; para el tratamiento de los datos se utilizó un análisis temático. Los resultados muestran incidentes asociados a desconsideración docente, exclusión territorial, prácticas no diversificadas y disputas
\end{abstract}

Palabras clave: inclusión, programas de integración escolar, participación, incidentes críticos, estudiantes.

* Psicólogo, magíster en Desarrollo Cognitivo, doctor (c) en Educación, Centro de Desarrollo Cognitivo, Universidad Diego Portales. ORCID: http://orcid.org/0000-00022756-1831. Contacto: ignacio.figueroa@mail.udp.cl

** Educadora Diferencial, magíster en Desarrollo Cognitivo, Centro de Desarrollo Cognitivo, Universidad Diego Portales. ORCID: https://orcid.org/0000-0002-9363-4955. Contacto: nataly.arevalo@mail.udp.cl

*** Psicólogo, magíster en Comunicación Política, Centro de Desarrollo Cognitivo, Universidad Diego Portales. ORCID: https://orcid.org/0000-0001-7171-9686. Contacto: cristopher.yanezu@mail.udp.cl

**** Educadora diferencial, magíster en Desarrollo Cognitivo, Centro de Desarrollo Cognitivo, Universidad Diego Portales. ORCID: https://orcid.org/0000-0002-6703-6652. Contacto: fernanda.munoz@mail.udp.cl

Los autores agradecen la colaboración de Yisví Paz en la elaboración de este artículo. 
entre estudiantes, generando emociones como rabia, vergüenza y tristeza.

\begin{abstract}
This article describes and analyzes serious incidents that have occurred in the classroom and which were reported by 24 students from seventh grade to the third year of secondary school, that were part of integration programs in schools in vulnerable situations in Santiago and La Serena, Chile. This is a qualitative research project with a narrative design and descriptive scope. The information was collected through reports of critical incidents, drawings and semi-structured interviews. A thematic analysis method was used to process the data. The results show incidents of teacher negligence, geographical exclusion, nondiversified practices and conflicts between students, generating emotions such as anger, shame and sadness.
\end{abstract}

\section{RESUMO}

Este artigo descreve e analisa os incidentes críticos relatados por 24 estudantes do $7^{\circ}$ ano do ensino fundamental ao $3^{\circ}$ ano do ensino médio, pertencentes a programas de integração de escolas em situação de vulnerabilidade de Santiago e La Serena, no Chile. Trata-se de uma pesquisa qualitativa de desenho narrativo e de escopo descritivo. As informações foram coletadas através de pautas de incidentes críticos, desenhos e entrevistas semiestruturadas. Uma análise temática foi utilizada para o processamento dos dados. Os resultados mostram incidentes associados à desconsideração dos professores, exclusão territorial, práticas não diversificadas e disputas entre estudantes, gerando emoções como raiva, vergonha e tristeza.
Keywords: inclusion, school integration programs, participation, critical incidents, students.

Palavras-chave: inclusão, programas de integração escolar, participação, incidentes críticos, estudantes. 


\section{Introducción}

Si bien en Chile durante el último tiempo ha existido un énfasis por abordar las desigualdades del sistema educativo (Gárate, 2019), tal como ocurrió con la Ley de Inclusión Escolar (Ley 20.845, 2015), aún existen una serie de barreras vinculadas a la hibridez de la política pública, que oscila entre un enfoque inclusivo y uno centrado en la integración (López et al., 2018). Lo anterior se plasma con mayor claridad en el desarrollo del Programa de Integración Escolar (PIE), en el que opera simultáneamente una mirada clínico-rehabilitadora y centrada en el déficit individual (Peña, 2013), y otra que vela por el ejercicio del derecho a la educación y participación (López, Morales, Rojas y Pérez, 2014). Esto produce que los estudiantes etiquetados con necesidades educativas especiales (NEE) asociadas a discapacidad sean constantemente posicionados en una situación ambigua de segregación e inclusión (Muñoz-Abarca, Figueroa y Yañez-Urbina, 2020).

En este sentido, como señalan Kozleski, Artiles y Waitoller (2014), el progreso en temáticas de inclusión es complejo y siempre local, ya que está conectado a sistemas de marginación profundamente arraigados, que operan clasificando y segregando al estudiantado a partir de una serie de marcadores de diferencia, entre ellos la raza, el género, la etnia y su capacidad cognitiva. Estos sistemas de marginación, al ingresar en la gramática escolar (Tyack y Cuban 2001), producen prácticas de opresión y silenciamiento que afectan la participación del estudiantado (Susinos y Ceballos, 2012), dinámica que ha sido denominada en la literatura como "inclusión excluyente" (Grinberg, 2015; Veiga-Neto, 2001) o "simulacro de inclusión" (Villalobos et al., 2015).

De acuerdo con Booth y Ainscow (2015), uno de los desafíos que se plantea a la educación es lograr reconocer las experiencias de todos los miembros de la institución desde la dimensión de la participación, en términos extendidos más allá del aula para abarcar toda la vida escolar. Sin embargo, las investigaciones en torno a la participación en las escuelas chilenas dan cuenta de la existencia de una participación tutelada, instrumental y utilitarista, guiada siempre por los adultos y produciendo una subjetividad estudiantil dependiente y pasiva (Ascorra, 2010; Ascorra, López y Urbina, 2017). 
En esta línea, existe evidencia que apunta a la relación entre los factores específicos (sectores de aprendizaje, momento de la clase, entre otros) del ambiente escolar en el que el estudiantado interactúa y su percepción de la participación dentro de la comunidad escolar (Erickson, Welander \& Granlund, 2007). Tomando en consideración el caso de estudiantes etiquetados con NEE, la literatura indica que, para lograr la participación activa, es necesario promover interacciones y relaciones significativas que deriven en experiencias positivas y atractivas en la escuela (Almqvist \& Granlund, 2005; Vetoniemi \& Kärnä, 2019), lo que tiene repercusiones en el bienestar subjetivo de estudiantes y sus familias (Galarce, Pérez y Sirlopú, 2020).

Por lo tanto, la presente investigación explora las experiencias de participación de estudiantes etiquetados con NEE, con un énfasis en aquellas situaciones o acontecimientos conflictivos que irrumpen en la vida escolar, poniendo en crisis su identidad y abriendo una fisura que nos permite acceder a sus representaciones de mundo (Valdés y Monereo, 2012). Nuestra finalidad es dar cuenta de cómo vivencian la participación desde sus propias voces, desarrollando la capacidad de evaluar y denunciar las barreras a la inclusión y promover los esfuerzos para la mejora educativa (Figueroa y Yáñez-Urbina, 2020; YáñezUrbina, Figueroa, Soto y Sciolla, 2018).

\section{Programa de Integración Escolar}

En la historia del sistema educacional chileno era posible evidenciar la existencia de una política de "educación especial" y de una "educación regular" que promovían activamente la segregación estudiantil (Ramos, 2014). Con el tiempo, se fue instalando la necesidad de incluir la diversidad al interior del aula regular, para garantizar el acceso igualitario a todo tipo de estudiantes, cuestión que no dejó de lado la impronta cultural de la segregación (López et al., 2018). En este escenario se instalan los PIE, como una estrategia de la atención educativa diferenciada para estudiantes que sean evaluados con alguna discapacidad de tipo intelectual, sensorial, relacional y/o comunicacional, o bien dificultades de aprendizaje (Ministerio de Educación [MINEDUC], 2010). Todos ellos etiquetados bajo el rótulo de NEE desde una lógica del déficit (Apablaza, 2018), para referir a una individualización de las problemáticas de aprendizaje que escapan de los recursos metodológicos habitualmente empleados por los docentes (Esparza, 2019). 
El estudiantado ingresa al programa por medio de una evaluación diagnóstica realizada por especialistas certificados por el MINEDUC, quienes establecen un diagnóstico basado en el tipo de necesidades educativas requeridas y en cuanto a si son permanentes [NEEP] o transitorias [NEET]. En ambos casos, los establecimientos reciben una subvención especial por cada estudiante inscrito en el PIE (Decreto 170, 2009).

Si bien los programas de integración escolar nacen para responder a las necesidades educativas del estudiantado, siendo denominados por el MINEDUC (2013) como una "estrategia inclusiva”, su actuar se inscribe bajo una lógica biomédica y asistencialista que aborda las problemáticas desde un foco individual y centrado en el déficit del estudiantado (Apablaza, 2018). Las consecuencias prácticas de este abordaje es la producción del estudiantado como sujeto pasivo y con bajas expectativas académicas en su desarrollo futuro (López et al., 2014). Asimismo, refuerza la segregación y la discriminación al interior de la escuela, dejando de lado aspectos culturales, sociales y prácticas educacionales que mantienen las brechas entre el estudiantado, y limitando la posibilidad de avanzar en prácticas más inclusivas y participativas (Damm, 2014; Gómez, 2014; Ramos, 2014).

\section{Inclusión educativa, voz del estudiantado y participación}

La inclusión educativa es aquel proceso de mejora continua del sistema educativo, con el propósito de eliminar las barreras al aprendizaje y la participación a las que se ve enfrentado el estudiantado (Booth y Ainscow, 2015). Abordar este enfoque resulta un gran desafío para las comunidades educativas; no obstante, representa un gran potencial para dar cuenta de cómo dichas barreras responden a criterios culturales y sociales de construcción de la diferencia entre grupos (Figueroa, Soto y Sciolla, 2016; Kozleski, Ariles \& Waitoller, 2014).

Desde una perspectiva crítica de la inclusión educativa, resulta necesario un abordaje que propicie la construcción de espacios de escucha de la voz del estudiantado, lo que implica considerar a niños, niñas y jóvenes como sujetos creadores de sus procesos educativos (Susinos y Rodríguez, 2011; Vergara, Peña, Chávez y Vergara, 2015; Sañudo y Susinos, 2018). Este desafío se instala en un horizonte de orden 
ético-político, que tiene como punto de partida las interrupciones que esta multiplicidad de voces genera en el espacio educativo (Messiou \& Ainscow, 2020), por medio del cuestionamiento sobre quién puede hablar o producir un conocimiento válido sobre la escuela, sus necesidades y proyecciones futuras (Figueroa y Yáñez-Urbina, 2020; YáñezUrbina et al., 2018).

Lo anterior implica procesos de indagación y reflexión en los que emerja una interpelación crítica a la perpetuación de una cultura profesionalista y adultocéntrica (Duarte, 2012; Escobedo, Sales y Traver, 2017) que invalida el actuar del estudiantado y su autonomía para intervenir activa y progresivamente en procesos de transformación social (Gascón y Godoy, 2015; Vergara et al., 2015). De esta forma, emerge la necesidad de abrir espacios intersticiales de diálogo y encuentro entre la multiplicidad de formas de entender el mundo que cohabitan en la escuela (Figueroa y Yáñez-Urbina, 2020) y que han sido desplazadas para el caso de estudiantes etiquetados con NEE (Muñoz-Abarca et al., 2020).

No obstante, estudios han destacado las problemáticas en los procesos de desarrollo escolar inclusivo para abrir espacios a la participación estudiantil (Figueroa y Muñoz, 2015; Figueroa et al., 2016), cuestión que ha derivado en la necesidad de desarrollar estrategias para fomentarla (Yáñez-Urbina et al., 2018; Figueroa et al., 2020), puesto que consiste en un derecho establecido jurídicamente y constituye un excelente medio para la formación de la ciudadanía en los valores democráticos que son claves para la vida en comunidad (Trilla y Novella, 2011).

De acuerdo con Ferreiro (2009), la participación en el aula implica la colaboración activa en el planeamiento, gestión, desarrollo y evaluación del proceso educativo en los distintos niveles de concreción curricular, propiciando el proceso de aprendizaje cuya finalidad es conseguir su autoformación educativa. Pero, generalmente, dentro del aula la participación estudiantil resulta escasa y restringida, sobre todo por el formato jerárquico tradicional de enseñanza, dominado por pautas unilaterales instauradas por los docentes (Urbina, Ipinza y Gutiérrez, 2020). Por lo tanto, es necesario abordar esta situación y dar mayor cabida a la participación en el aula para visualizar la realidad de los estudiantes dentro del contexto escolar, lo que permitiría definir 
con claridad lo que piensan y esperan de su experiencia en el establecimiento educacional (Prieto, 2005).

En este sentido, Hidalgo y Perinés (2018) concluyen que, a pesar de que el profesorado declara fomentar la participación estudiantil, no despliega estrategias para estimular la colaboración en la selección de contenidos, determinación de estrategias didácticas o de evaluación. Cuestión de gran interés, ya que autores como Prieto (2005) destacan el protagonismo del rol docente en la construcción de un ambiente que propicie la participación y, con ello, la construcción de una identidad de aprendiz legítima, es decir, como un sujeto activo e implicado en sus propios procesos de aprendizaje (Julio, 2017).

\section{Incidentes críticos}

Una estrategia privilegiada para brindar espacios de escucha a la voz del estudiantado y gatillar su participación corresponde a la propuesta del análisis de incidentes críticos (IC). Por IC se entiende un suceso de la vida cotidiana que causa perplejidad, por ser desafiante en términos intrapersonales e interpersonales (Monereo y Monte, 2011). Esta clase de situaciones requieren de respuestas rápidas, por lo que suelen requerir reacciones innovadoras (Almendro y Costa, 2006), que ponen en juego una serie de competencias sociales y emocionales para poder enfrentarlas y gatillar procesos de reflexión continua sobre el actuar (Monereo, 2010).

Según Vallejos (2013), la técnica de análisis de IC consiste en una descripción secuencial y detallada de lo ocurrido para su ulterior descomposición, tanto en aspectos emocionales, actitudinales y en las tensiones que presentó, como en las posibilidades de actuación que existieron en su momento. La idea de esta estrategia consiste en generar instancias de reflexión, individuales o colectivas, acerca de una situación de difícil solución, para poder abordarla de manera consciente en un futuro.

Investigaciones señalan que es inevitable la existencia de incidentes críticos en los contextos educativos, sin importar la dependencia educacional ni el ciclo educativo (Bilbao y Monereo, 2011), siendo necesario el trabajo reflexivo para comprender los acontecimientos y redireccionar las prácticas que impactan a los miembros del establecimiento escolar. 
Existe un marcado privilegio de la investigación de los incidentes críticos en educación desde la perspectiva de los docentes (Nail, Gajardo y Muñoz, 2012; Gajardo, Ulloa y Nail, 2017; Bilbao y Monereo, 2011; Valdés y Monereo, 2012; Figueroa, Sepúlveda, Soto y Yañez-Urbina, 2020), mientras que aquellos estudios que abordan la voz del estudiantado suelen enfocarse en educación superior (Click, 2018; Sevilla \& Gamboa, 2016) y, cuando focalizan en educación escolar, muestran un marcado carácter instrumental orientado a la adaptación de formatos de clases y mejora del rendimiento académico (Hong \& Song, 2020; Kaukko \& Wilkson, 2020; Nyman, Parisod, Axelin \& Salantera, 2019).

En contrapartida con esta tendencia, Paulin et al. (2011) realizaron una exploración con estudiantes de educación secundaria en Argentina, puntualizando en la existencia de dinámicas en las que los docentes no responden a sus inquietudes e interrogantes cuando imparten una clase. Es necesario, entonces, desarrollar investigaciones que adopten la perspectiva del análisis de IC y que permitan generar espacios de escucha de la voz del estudiantado para el diagnóstico de aquellas barreras al aprendizaje y la participación a las cuales se enfrentan en las aulas chilenas.

\section{Método}

El estudio responde a un enfoque cualitativo de investigación con un alcance descriptivo, pues se focaliza en una investigación del mundo social desde la premisa que éste es construido por medio de significados y símbolos (Salgado, 2007). Siguiendo esta línea, se procedió por medio de un diseño narrativo como una estrategia para profundizar en el mundo identitario, construir significados del saber práctico de los actores sociales, centrado en sus vidas cotidianas, relaciones, reconocimientos personales y culturales (Bolívar y Domingo, 2006), lo que se constituye como una investigación inclusiva al constituir un espacio de escucha de la voz del estudiantado (Susinos y Ceballos, 2012). De tal manera, se enfatizó en la narración o historias elaboradas por los participantes acerca de sus experiencias de vida (Salgado, 2007), con un énfasis en un abordaje de tópicos (Martens, 2010) tales como lo son los IC a propósito de su participación en aula.

Por lo tanto, la selección de participantes se dio a través de un muestreo por conveniencia, priorizando aquellos estudiantes usuarios 
del PIE etiquetados con alguna NEE de tres escuelas municipales de la ciudad de La Serena, en la Región de Coquimbo, y una escuela particular subvencionada de la ciudad de Santiago, en la Región Metropolitana, entre los años 2017-2019. Estas escuelas tienen en común el hecho de que trabajan con la política pública de integración educativa y están ubicados en contextos de vulnerabilidad escolar, resultando accesibles para el equipo investigador. En tal sentido, la muestra del estudio se conformó por 24 estudiantes (13 de género femenino y 11 de género masculino) entre séptimo año de enseñanza básica y tercero medio, de los cuales 20 fueron etiquetados con NEET y cuatro con NEEP, de acuerdo con la normativa de integración. En el caso de las NEET aparecen los diagnósticos de Déficit de Atención (DA) y de Funcionamiento Intelectual Límite (FIL), mientras que en la NEEP existen estudiantes diagnosticados con Discapacidad Intelectual Leve (DIL).

Tabla $\mathrm{N}^{\circ} 1$

\begin{tabular}{lccc}
\hline CURSO & NEET DA & NEET FIL & NEEP DIL \\
\hline Colegio N 1 La Serena & 5 & 1 & 0 \\
\hline Colegio N² La Serena & 7 & 1 & 0 \\
\hline Colegio N³ La Serena & 5 & 1 & 0 \\
\hline Colegio N ${ }^{\circ}$ Santiago & 0 & 0 & 4 \\
\hline TOTAL & 17 & 3 & 4 \\
\hline
\end{tabular}

Fuente: Elaboración propia.

Las técnicas de producción de información empleadas fueron una entrevista semiestructurada, con un enfoque narrativo, es decir, el desarrollo de una dinámica de preguntas-respuestas enfocadas en producir una narración desde la experiencia de los participantes, y que contó con una estructuración flexible elaborada previamente (Alheit, 2012), con base en las dimensiones del análisis de IC (descripción de la situación, emociones, actuación y reflexión sobre la situación) (Nail et al., 2011); complementada con la confección de un dibujo como una herramienta que permite no solamente gatillar un encuentro para el intercambio comunicacional (Banks, 2010), sino también facilitar el ingreso a un universo de significados desde los códigos propios del estudiantado.

Posteriormente, el material producido fue sometido a un análisis narrativo temático (Riessman, 2008), construyendo categorías analíticas 
emergentes sobre los IC reportados por los estudiantes en las entrevistas y sus dibujos, lo cual fue llevado a cabo por medio de un procedimiento comparativo constante. En relación con los aspectos éticos de la investigación, se solicitó autorización al director de cada establecimiento y se entregó un consentimiento informado a los apoderados de los estudiantes participantes. Además, se comunicó al estudiantado sobre la investigación, los propósitos y las implicancias de esta, gestionando un asentimiento informado. Sumado a lo anterior, se generó un espacio para responder dudas del estudiantado, clarificando siempre que su participación en la investigación era voluntaria y confidencial.

\section{Resultados}

A continuación se presentan los resultados del análisis de IC, los cuales se expresan en cuatro categorías.

\section{Desconsideración del estudiantado por parte de sus docentes}

Corresponde a aquellos incidentes que describen actitudes de indiferencia de parte de los docentes hacia el estudiantado, reflejadas en prácticas de invisibilización, restricción del diálogo entre estudiantes y falta de accesibilidad ante dudas e interrogantes del estudiantado. Frente a esta clase de incidentes, los y las estudiantes sienten que no son atendidos por parte del equipo docente, quedando expuestas sus necesidades y demandas contextuales, dando como resultado una limitación a su participación.

A través de estos incidentes, los estudiantes reportan experiencias en las que su solicitud de ayuda no pudo ser acogida ni abordada. En esta línea, se plantean dinámicas de invisibilización del lugar que ocupan al interior del aula regular, situación que no solamente dificulta su participación activa y a la par del resto de sus compañeros, sino que también genera malestar y retraimiento, por ser considerado como un estudiante que no puede otorgar respuestas, tal como se aprecia en el siguiente extracto:

Estábamos viendo el reino de los animales en ciencias naturales, y la profesora hace una pregunta que yo me la sabía, levanté la mano para responderla, la profesora me mira, pero le da la palabra a otra compañera sin tomarme en cuenta. (Estudiante 16) 
Por otra parte, el estudiantado reporta desconsideración a partir de juicios apriorísticos de los docentes acerca de situaciones de aula en las que existen intercambios entre estudiantes. Estas instancias regularmente son interpretadas por los docentes como distracciones o pérdida de tiempo, desincentivando la colaboración y el trabajo de equipo entre pares. Ante esto, se destaca que, en estas situaciones, el estudiantado percibe que no se acogen sus argumentos y perspectivas.

En la clase de Historia, cuando estábamos haciendo una tarea de la página del libro (del estudiante), me levanté de mi asiento para preguntarle a un compañero una duda que tenía sobre la tarea. Mientras mi compañero me estaba explicando, la profesora nos reta a ambos por creer que estábamos solo conversando, y porque yo me había levantado de mi puesto sin permiso, e intentamos explicar que era una ayuda, pero sin embargo me tuve que ir a sentar a mi asiento. (Estudiante 3 )

Otra manera en que se expresa la desconsideración es cuando los y las estudiantes no obtienen respuestas satisfactorias de parte de los docentes, al presentar dudas ante determinados temas. Este tipo de situaciones se vincula con contenidos de aprendizaje que resultan complejos y desafiantes para el estudiantado. Por ejemplo, en la Ilustración 1 se relata un IC en el que un estudiante se ve enfrentado a la resolución de un ejercicio matemático, resultando expuesto ante la profesora y todos sus compañeros.
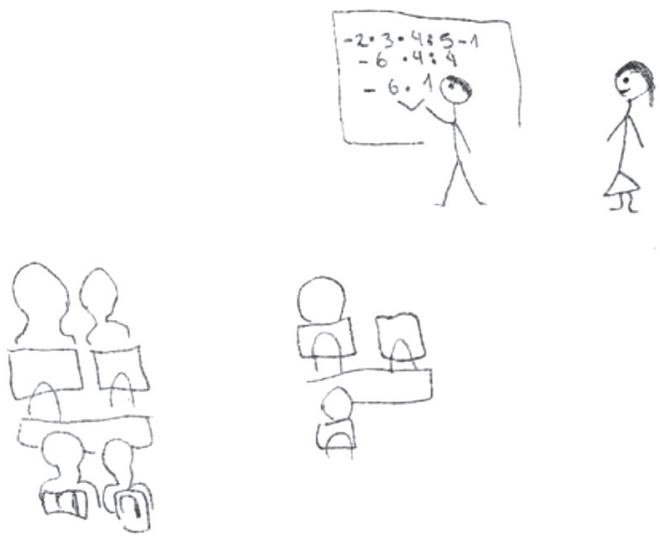

Ilustración 1: "En frente de todos"

Fuente: Dibujo estudiante 22. 
Este tipo de incidentes dan cuenta de una dinámica de aula en la que se producen brechas de aprendizajes entre el estudiantado que no logra resolver el ejercicio dispuesto por la docente. Adicionalmente a la situación de exposición, estos eventos se acompañan de una sanción implícita, en el sentido que la incapacidad de dar una respuesta satisfactoria culmina con la orden de volver al asiento, sin una explicación de cómo realizar la tarea, perpetuando un sentimiento de temor y vergüenza que posteriormente influye de manera negativa en la participación del estudiante.

Estábamos en la clase de matemática y la profesora me hizo salir a la pizarra para hacer un ejercicio matemático sobre las potencias. Le dije a la profesora que no sabía cómo hacerlo y me dijo que me fuera a sentar, yo le hice caso y me fui a sentar, pero con la duda de cómo se hacía el ejercicio. Al final, no supe cómo hacerlo. (Estudiante 4)

\section{Exclusión territorial: los estudiantes PIE al aula de recursos}

Esta categoría se relaciona con la salida de los estudiantes del aula regular hacia el aula de recursos. Cuestión que, a juicio del estudiantado, implica tanto un desarraigo de su espacio de experiencias cotidianas como de la misma oportunidad de resolver problemas y mejorar sus procesos de enseñanza y aprendizaje.

Frente a esta clase de IC, se logra evidenciar una relación ambivalente de la acción de "ser sacado" del aula. Ello debido a que, si bien el tránsito hacia el aula de recursos consiste en una serie de apoyos pedagógicos brindados por parte de los miembros del equipo del PIE, esta misma acción de salir de las clases conlleva perder acceso a parte del curriculum. Por lo tanto, no solamente se experimenta un proceso de segregación, en términos de su necesidad de recibir atención diferenciada, sino que también de su capacidad de acceder a los mismos contenidos que el resto de sus pares.

En la clase de inglés llega la psicóloga a buscarme (para ir a trabajar en la sala de integración). Yo no quiero salir, porque me voy a atrasar en la materia y pienso que después voy a andar "perdido en clases". (Estudiante 2)

Por otro lado, este tipo de eventos no solamente afecta en términos de acceso al curriculum, sino que además en desmedro de las expe- 
riencias cotidianas que pueden ser vividas en el aula en momentos de compartir, tanto con los docentes como con sus compañeros, situación que produce un malestar asociado a la privación de experiencias gratificantes de socialización y de encuentro con el resto.

Estábamos en la hora de matemática y de repente llega la profesora Katy (educadora diferencial) a buscarme para ir a trabajar con ella a su sala (aula de recursos). Yo no quise salir porque estaba muy entretenida la clase, pero igual tuve que salir de la sala y perderme la clase. (Estudiante 9)

Finalmente, existen ocasiones en las cuales la salida al aula de recursos posibilita la resolución parcial de incidentes prácticos del aula. Por ejemplo, la Ilustración 2, elaborada por el Estudiante 22, plasma el momento en el cual es interrogado por parte del docente, al no contar con los materiales necesarios para desarrollar la actividad prevista en la planificación de la clase. Ante esta situación, el estudiante abandona la sala para dirigirse al aula de recursos, en donde sí puede contar con materiales para realizar la tarea, pero siendo de manera implícita expulsado del aula regular.

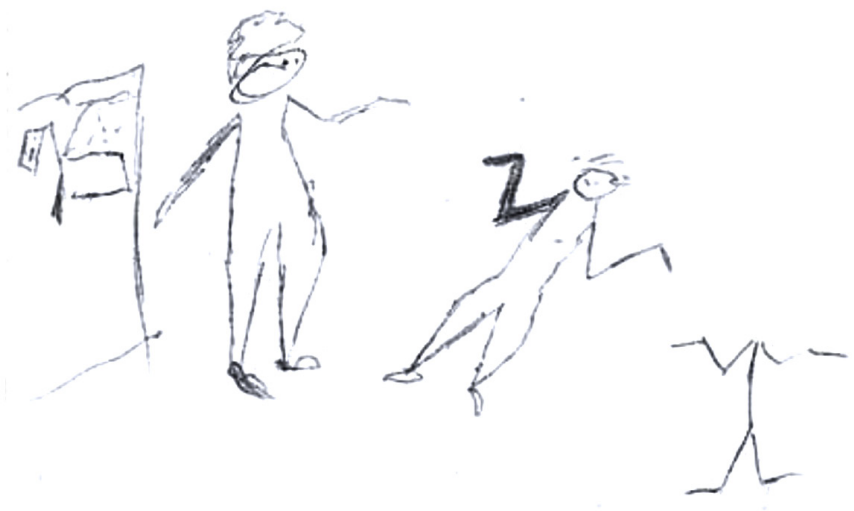

Ilustración 2: “¿Dónde están tus materiales?”

Fuente: Dibujo Estudiante 21.

\section{Prácticas pedagógicas no sensibles a la diversidad}

Otro tipo de incidentes reportados consisten en aquellas situaciones que develan prácticas pedagógicas que no responden a las necesida- 
des y características personales del estudiantado, cuestión que se resume en una falta de diversificación de estrategias pedagógicas para el abordaje de la diversidad en el aula.

De un lado, estas situaciones se plasman en una falta de adaptación de los instrumentos de evaluación, para permitir el acceso del estudiantado a la misma actividad y como condición mínima para el cumplimiento de la tarea. El incidente reportado por el Estudiante 5 es esclarecedor al respecto, puesto que consiste en una adaptación curricular consensuada previamente y que, al no ser tomada en cuenta al momento de la elaboración de la evaluación, culmina con la segregación de la experiencia pedagógica del estudiante, produciendo una sensación de malestar subjetivo.

Estábamos en la clase de lenguaje haciendo una prueba y cuando comienzo a realizarla me doy cuenta de que a la profesora se le olvidó (yo creo) que debe hacerme las pruebas con la letra número 16 (por mi problema de visión) y me dio vergüenza decirle. Traté de hacer lo que más pude, pero con mucha dificultad, al final no pude finalizar como yo quería. (Estudiante 5)

Por otro lado, también es posible evidenciar esta clase de incidentes en las dinámicas cotidianas al interior del aula. A partir del dibujo de la Estudiante 24 (Ilustración 3) se narra una clase en la que la docente imparte su asignatura dictando los contenidos para que los estudiantes los copien en sus cuadernos. Producto de esta práctica, y asociado a sus características personales, la estudiante no logra desempeñarse a la misma velocidad que avanza la docente, situación que le produce vergüenza e impide que reporte su dificultad, ocasionando que prefiera perder contenidos antes que exponerse.

Ahí estamos en historia... ahí está la $A$, la B estoy yo... ahí está la profe... ahi dictan, dictan, escriben y yo me pierdo... Yo conversaba con mis compañeras ¿cómo era la palabra? Yo pensaba decirle a la profe si puede dictar lo que dijo que me perdi, pero no lo hice igual... como estaba ocupada. (Estudiante 24) 


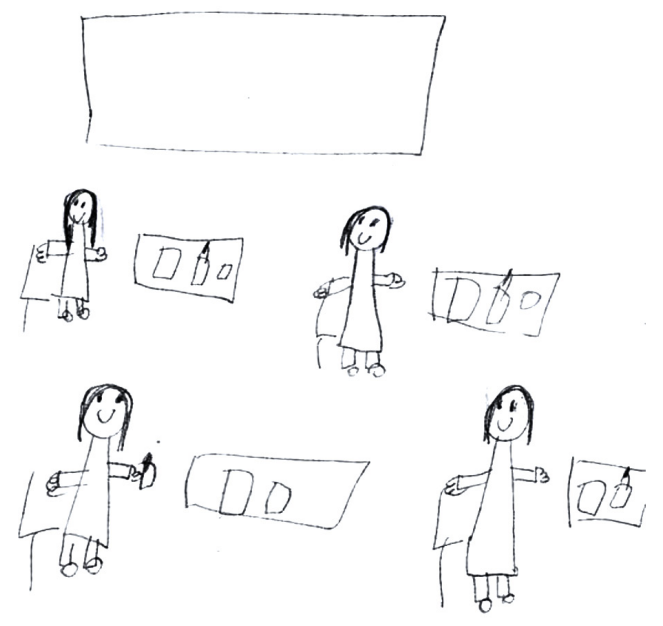

Ilustración 3: "El dictado"

Fuente: Dibujo Estudiante 24.

Asimismo, ocurre una tercera situación en la cual el incidente corresponde a las ocasiones en las que el estudiantado se ve sorprendido por evaluaciones adaptadas que no le han sido informadas previamente. Este fenómeno está asociado no solo con el impacto, sino que también con la incertidumbre a la cual se ven expuestos, por enterarse a través de mecanismos no oficiales, por no entender a qué se debe y por la falta de una mediación que posibilite comprender la intención detrás de la medida propuesta por el docente, reproduciendo una dinámica de estigmatización al percibir diferencias con el resto de sus compañeros.

Al terminar la prueba de matemática nos pusimos a comentar la prueba con mis compañeras y me di cuenta en ese momento que mi prueba era diferente (por la cantidad de preguntas) a la de mis compañeras. Le pregunto de inmediato al profesor por qué mi prueba tenía menos preguntas y me respondió solo que mi prueba era diferente. Yo no le dije nada más, pero luego lo comenté con mis compañeras. (Estudiante 14)

\section{Disputas entre estudiantes}

Un último tipo de incidente considera aquellas situaciones en las que, debido a desacuerdos o conflictos entre estudiantes, se genera des- 
atención o pérdida del proceso de aprendizaje. En un primer grupo de incidentes se rescatan aquellas dificultades vivenciadas por el estudiante para integrarse a grupos de trabajo en el contexto de una tarea $o$ actividad pedagógica. Tal como se aprecia en el extracto del Estudiante 22, estos eventos no solamente están asociados con la necesidad de participar en el aula, las dificultades encontradas en aquel momento y los mecanismos de resolución, sino también con la vivencia de procesos de desconsideración a los cuales se encuentra expuesto por parte de sus pares.

En lenguaje hay que hacer grupos de cuatro o cinco integrantes y yo siempre me voy a uno y le pregunto al A si estaba lleno su grupo, si me podía ingresar a mí, y me dijo que no... como siempre tiene amigos, asíque me fui a otro grupo y le pregunté a mis compañeras de la feria científica si podía hacer grupo con ellas y me dicen que sí. Yo me acerqué al grupo, pero me ignoró... me ignoraron no más, no dijeron na' ellos siguieron riéndose, les hablé fuerte para que me escucharan, pero estaban ignorándome, así que decidí cambiarme a otro grupo porque nadie me hablaba, y el otro grupo me habló. (Estudiante 22)

Profundizando sobre esta misma narrativa, la Ilustración 4 da cuenta de cómo el estudiante percibe esta dinámica de invisibilización y su toma de acción frente a la desconsideración de sus pares al ignorarlo, cuestión que desarrolla por medio de una fábula visible que le permite dar cuenta de las estrategias desplegadas para su resolución, por medio del dibujo de un revólver para defenderse:

Voy a hacer lo último por si me sale... listo, una pistola por si acaso me atacan, si me atacan salgo corriendo (...) y le aviso al inspector... la pistola es para que se asusten y van a estar enojados. (Estudiante 22) 


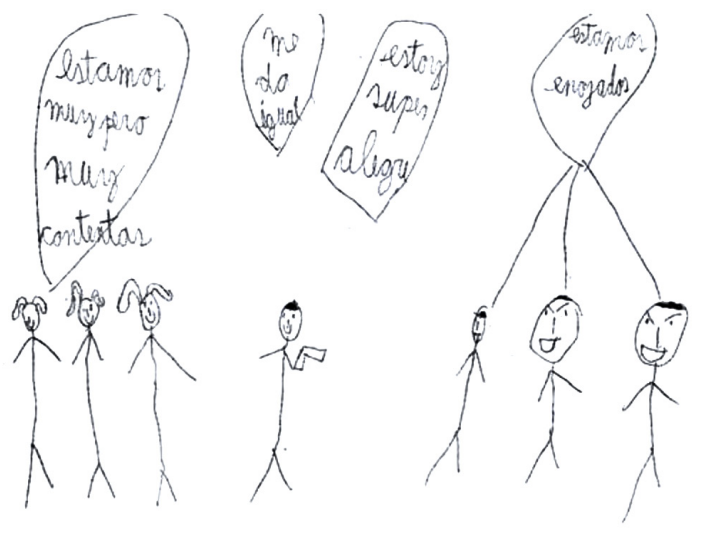

Ilustración 4: "La pistola por si me atacan" Fuente: Dibujo Estudiante 22.

Por último, este mismo tipo de disputas y desconsideraciones se manifiestan en prácticas disruptivas que derivan en situaciones de burla. La narración del Estudiante 19 da cuenta de un episodio en el cual un compañero genera una situación vergonzosa, que coincide con un momento en el cual la docente no se percata, ante lo cual el estudiante debe recurrir al equipo del PIE como un mecanismo de resguardo.

Cuando terminamos de hacer la prueba de ciencias naturales, un compañero me quitó el celular y empezó a mandar fotos y cosas "cochinas" a los demás niños. La profesora no se dio cuenta de eso. (Estudiante 19)

\section{Discusiones y Conclusiones}

A la luz de los resultados respecto de los tipos de incidentes críticos vinculados a la participación del estudiantado, se puede apreciar la existencia de cinco grandes nudos críticos referidos a (1) la desconsideración por parte de docentes, (2) la falta de implementación de adecuaciones requeridas, (3) la implementación de adecuaciones sin informarlas al estudiantado, (4) la exclusión del aula regular hacia el aula de recursos, y (5) la conflictividad y desconsideración que se da entre pares. Cada una de ellas despliega una serie de dinámicas y emociones que posibilitan realizar un encadenamiento de la trayectoria 
que toman las narraciones de los estudiantes, el cual se presenta en la Ilustración 5.

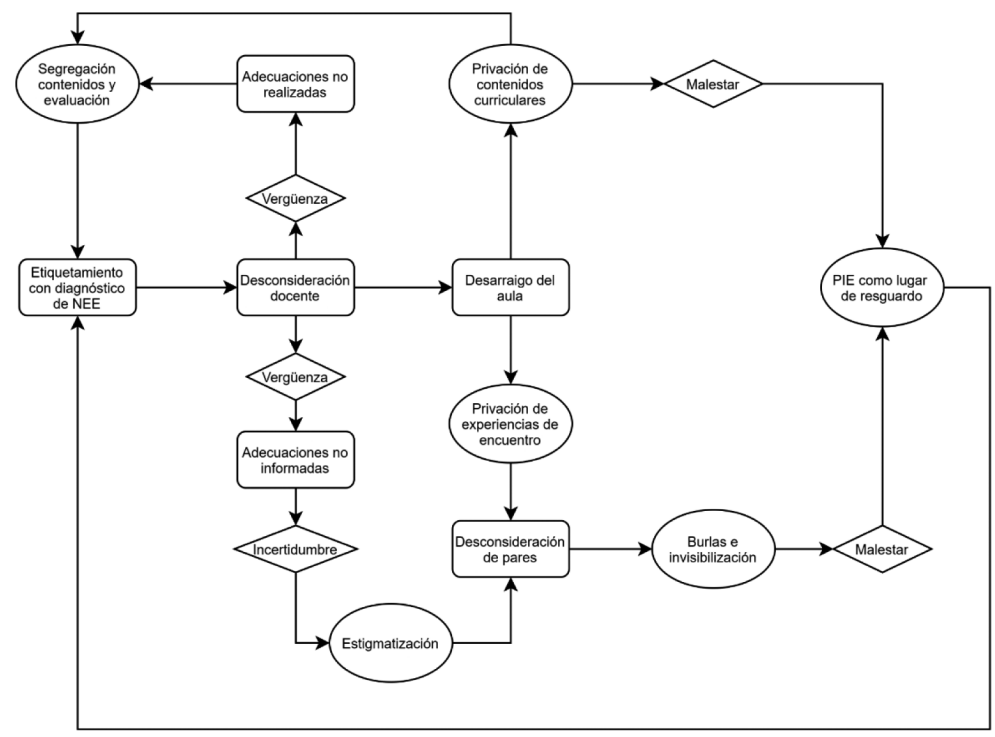

Ilustración 5: Trayectoria de las narraciones estudiantiles en el PIE Fuente: Elaboración propia.

Tal como se puede apreciar, los incidentes críticos reportados van más allá de un acto situacional o circunstancial que aparece de forma aislada a través del ciclo escolar. Al contrario, responden más bien a una lógica sistemática en la que el estudiantado, etiquetado con algún diagnóstico de NEE, es inscrito en la reproducción de un programa de apoyo de corte asistencialista y centrado en el déficit (Apablaza, 2018; López et al., 2014; Muñoz-Abarca et al., 2020; Peña, 2013). Esto por medio de una individualización de la problemática, sin tomar en consideración la necesidad de desplegar recursos pedagógicos diversificados que atiendan a la heterogeneidad del aula (Esparza, 2019).

Lo curioso de la dinámica expuesta es que opera a contrapunto de las narrativas de los adultos que consideran al estudiantado como un agente pasivo y mero asimilador de las lógicas impuestas (Gascón y Godoy, 2015; Vergara et al., 2015; Julio, 2017). Como se aprecia, tanto los estudiantes etiquetados con un diagnóstico de NEE como sus pares participan activamente de la trayectoria. Los segundos, influenciados 
por una serie de dinámicas producidas por el abordaje del PIE, en el que se refuerza la estigmatización y la privación de experiencias de encuentro del estudiantado al que apoya, resultando una barrera para la apreciación y el respeto de la diferencia al interior del espacio educativo. Mientras que los primeros son llevados a encontrar en el PIE un espacio de resguardo ante una serie de emociones adversas y que impactan en su bienestar subjetivo al momento de habitar la escuela.

No obstante, esto no implica que el propio estudiantado sea la raíz de la problemática, sino que se encuentra guiado por una dinámica cultural profesionalista y adultocéntrica que opera en la escuela y en el PIE, la cual tiende al silenciamiento de la diferencia y a propiciar la primacía de la validación de los estudiantes por medio de su rendimiento académico (Duarte, 2012; Escobedo et al., 2017). De esta manera, según Muñoz-Abarca et al. (2020), en el PIE se produce una doble subalternización del estudiante etiquetado con un diagnóstico de NEE asociado a discapacidad, es decir, primero como un estudiante y luego como un estudiante anormal.

Por lo tanto, se evidencia que la participación en el aula es gestada por el mundo adulto para encaminarla hacia la producción de una subjetividad estudiantil dependiente y pasiva (Ascorra, 2010; Ascorra et al., 2017). Siendo así, cobran relevancia aquellos aspectos específicos del ambiente escolar en el que se desarrollan las interacciones en aula, para lograr una transformación de la percepción de participación del estudiantado dentro de la comunidad escolar (Almqvist et al., 2005; Erickson et al., 2007; Vetoniemi \& Kärnä, 2019; Willis et al., 2020) y una preocupación por el bienestar subjetivo (Galarce et al., 2020).

Por lo tanto, cabe preguntarse y especular, desde los aportes de la bibliografía especializada, acerca de posibles acciones que tiendan a tomar los incidentes del estudiantado como una fuente de información válida para la transformación de las dinámicas en el aula que operan a modo de una inclusión excluyente (Grinberg, 2015; Veiga-Neto, 2001) o simulacro de inclusión (Villalobos et al., 2015). De tal manera, estas experiencias pueden constituir insumo para la superación de las barreras a la inclusión (Booth y Ainscow, 2015), garantizando el ejercicio del derecho a la participación como un medio para la formación integral (Trilla y Novella, 2011). 
En primer lugar, destaca la necesidad de realizar un trabajo sobre la percepción de no ser escuchados que reportan los estudiantes en sus IC, y que repercute en la producción de vergüenza, malestar e incertidumbre. Un ejemplo de esto es la aplicación de una evaluación diferenciada, sin toma de conocimiento de parte del estudiante, práctica que refuerza una identidad de aprendiz pasivo, cuestión que, de acuerdo con Urbina, Ipinza y Gutiérrez (2020), requiere ser integrada, en tanto parte de las inquietudes del estudiantado, a las interacciones del aula, permitiendo asociar sus perspectivas con el contenido revisado en clases y dando pie a la coconstrucción de conocimiento desde una perspectiva democrática.

Sobre este mismo punto, tanto las políticas educativas, los establecimientos educativos como el cuerpo docente y de apoyo deben desarrollar estrategias y herramientas prácticas que permitan favorecer y fomentar que las perspectivas de niños, niñas y jóvenes sean consideradas y, de esa forma, brindar un soporte emocional que les permita no solamente enfrentar situaciones ansiógenas, sino que también desplegar una actuación orientada a su resolución efectiva. Lo que nos instala ante la necesidad de construir una identidad de aprendiz legítima (Julio, 2017), que supere la mirada del estudiante como agente pasivo que solamente debe seguir las instrucciones del docente (Ascorra, 2010). Por ejemplo, en dos de los casos, los estudiantes reconocen una buena resolución del incidente de parte de los docentes, lo que les lleva a sentir tranquilidad y alivio, situación que favorece la consiguiente recuperación de la posición de los estudiantes como productores de significado a través de vínculos de confianza y reconocimiento (Hart, Drummond \& McIntyre, 2013).

De esta manera, el abordaje de los incidentes críticos, puede aportar un contrapunto novedoso a la mirada del docente, permitiendo identificar "síntomas" de la práctica (Valdés y Monereo, 2012) y del despliegue concreto de las políticas educativas orientadas al abordaje a la diversidad; temas sobre los que es necesario reflexionar críticamente y con un alto sentido de agencia, incorporando activamente al estudiantado. En esta línea, acceder a las narrativas del aula (Vetoniemi \& Kärnä, 2019), a través de metodologías como la "indagación inclusiva" (Messiou \& Ainscow, 2020) y dispositivos que permitan construir espacios de diálogo entre docentes y estudiantes-investigadores (Figueroa y Yáñez-Urbina, 
2020), favorecen la producción de desacoplamientos (Urbina, Ipinza y Gutiérrez, 2020) e interrupciones reflexivas (Messiou \& Ainscow, 2020) en el quehacer pedagógico, abriendo espacios de innovación, participación y aprendizaje, por medio de la escucha, validación y reconocimiento de las voces y culturas estudiantiles.

\section{Referencias}

Alheit, P. (2012). La entrevista narrativa. Plumilla Educativa, 10(2), 1118. DOI: 10.30554/plumillaedu.10.84.2012.

Almendro, C. y Costa, A. (2006). Alerta roja: el incidente crítico, aprendiendo de nuestros errores. Tribuna docente, 8(4), 1-8. Recuperado de: https://www.uv.es/gibuv/IncidenteCritico.pdf.

Almqvist, L. y Granlund, M. (2005). Participation in school environment of children and youth with disabilities: a person-oriented approach. Scandinavian Journal of Psychology, (46), 305-314. DOI: 10.1111/j.1467-9450.2005.00460.x.

Apablaza, M. (2018).Inclusión escolar, marginación y apartheid ocupacional: Análisis de las políticas educativas Chilenas. Journal of Occupational Science, 25(4), 1-13. DOI: 10.1080/14427591.2018.1487260.

Ascorra, P. (2010). Aportes de las ciencias sociales al nuevo management. Psicoperspectivas, 9(1), 1-6. Recuperado de: https:// www.psicoperspectivas.cl/index.php/psicoperspectivas/article/viewFile/119/384.

Ascorra, P., López, V. y Urbina, C. (2016). Participación estudiantil en escuelas chilenas con buena y mala convivencia escolar (Students Participation in Chilean Schools with Good and Poor School Climate). Revista de Psicología, 25(2), 1-18. DOI: 10.5354/0719-0581.2016.44686.

Banks, M. (2010). Los datos visuales en investigación cualitativa. Madrid: Morata.

Bilbao, G. y Monereo, C. (2011). Identificación de incidentes críticos en maestros en ejercicio: Propuesta para la formación permanente. Revista Electrónica de Investigación Educativa, 13(1), 135-151. Recuperado de: http://www.scielo.org.mx/scielo. php?script=sci_arttext\&pid=S1607-40412011000100009.

Bolívar, A. y Domingo, J. (2006). La investigación biográfica y narrativa en Iberoamérica: campos de desarrollo y estado actual. 
Forum Qualitative Social Research, 7(4), art. 12. Recuperado de: https://www.qualitative-research.net/index.php/fqs/article/view/161/357.

Booth, T. y Ainscow. M. (2015). Guía para la Educación Inclusiva. Desarrollando el aprendizaje y la participación en los centros escolares. Madrid: OEI/FUHEM.

Click, A. (2018). International graduate students in the United States: Research processes and challenges. Library \& Information Science Research, 40(2), 153-162. DOI: 10.1016/j.lisr.2018.05.004

Damm, X. (2014). Representaciones y actitudes del profesorado frente a la integración de niños/as con necesidades educativas especiales al aula común. Revista latinoamericana de educación inclusiva, 3(1), 25-35. Recuperado de: http://www. rinace.net/rlei/numeros/vol3-numl/art2.html.

Decreto 170. (2009). Fija normas para determinar los alumnos con necesidades educativas especiales que serán beneficiarios de la subvenciones para educación especial. Biblioteca del Congreso Nacional. Recuperado de: https://www.leychile. $\mathrm{cl} / \mathrm{N}$ ? $\mathrm{i}=1012570 \& \mathrm{f}=2010-08-25 \& \mathrm{p}=$.

Duarte, C. (2012). Sociedades adultocéntricas: Sobre sus orígenes y reproducción. Última Década, 20(36), 99-125. DOI: https:// doi.org/10.4067/S0718-22362012000100005.

Eriksson, L., Welander, J. \& Granlund, M. (2007). Participation in everyday school activities for children with and without disabilities. Journal of Developmental and Physical Disabilities, 19(5), 485-502. DOI: 10.1007/s10882-007-9065-5.

Escobedo, P., Sales, A. y Traver, J. (2017). La voz del alumnado: su silencio y la cultura profesionalista. Educación XXI, 20(2), 299318. DOI: 10.5944/educxx1.19045.

Esparza, R. (2019). Perspectiva global de una educación inclusiva, en Chile y en los principales países sudamericanos. Educación, 25(1), 33-42. DOI: 10.33539/ducación.2019.v25n1.1765.

Ferreiro, R. (2009). Estrategias didácticas del aprendizaje cooperativo. México: Trillas.

Figueroa, I. y Muñoz, Y. (2014). La Guía para la Inclusión Educativa como herramienta de autoevaluación institucional: reporte de una experiencia. Revista Latinoamericana de Educación Inclusiva, 8(2), 179-198. Recuperado de: http://www.rinace. net/rlei/numeros/vol8-num2/art9.pdf. 
Figueroa, I., Sepúlveda, G., Soto, J. y Yáñez-Urbina, C. (2020). Coenseñanza entre docentes de educación general básica y educadoras diferenciales: incidentes críticos de la práctica colaborativa en programas de integración educativa. Pensamiento Educativo, 57(1), 1-15. DOI: 10.7764/PEL.57.1.2020.1

Figueroa, I., Soto, J. y Sciolla, B. (2016). Dinámicas de recepción, apropiación y contextualización del enfoque de la Guía para la inclusión educativa en escuelas municipales de una comuna de la Región Metropolitana. Pensamiento Educativo, 53(1), 1-13. DOI: 10.7764/pel.53.1.2016.4.

Figueroa, I. y Yáñez-Urbina, C. (2020). Voces y culturas estudiantiles en la escuela: una reflexión teórico-metodológica desde procesos de autoexploración de barreras a la inclusión educativa. Revista latinoamericana de educación inclusiva, 14(1), 109-126. DOI: 10.4067/S0718-73782020000100109.

Gajardo, J., Ulloa, J. y Nail, O. (2017). Análisis de incidentes críticos en la sala de clases: una oportunidad de aprendizaje profesional docente. Nota Técnica 7-2017. Recuperado de: https:// www.lidereseducativos.cl/wp-content/uploads/2017/12/ Nota-te\%CC\%81 cnica-N\%C2\%B07-L1.pdf.

Galarce, M., Pérez-Salas, C. y Sirlopú, D. (2020). Análisis comparativo de la participación escolar y bienestar subjetivo en estudiantes con y sin discapacidad en Chile. Psykhe, 29(2), 1-16. DOI: 10.7764/psykhe.29.1.1444.

Gárate, F. (2019). Inclusión educativa en Chile: un camino políticohistórico con ruta de empedrados, curvas y colinas. Revista Estudios en Educación, 2(2), 143-167. Recuperado de: http://ojs.umc.cl/index.php/estudioseneducacion/article/view/53.

Gascón, F. y Godoy, L. (2015). Presencia e in-diferencia. Por un estatuto visual de la niñez. Revista Latinoamericana de Ciencias Sociales, Niñez y Juventud, 13(2), 645-656. DOI: 10.11600/1692715x.1326093014.

Gómez, V. (2014). Análisis de la discapacidad desde una mirada crítica: las aportaciones de las teorías feministas. Estudios Pedagógicos, 15(2), 391-407. DOI: 10.4067/s071807052014000300023.

Grinberg, S. (2015). Dispositivos pedagógicos, gubernamentalidad y pobreza urbana en tiempos gerenciales: un estudio en la co- 
tidianeidad de las escuelas. Propuesta Educativa, 43(1), 123130. Recuperado de: http://ref.scielo.org/xv7kkf

Hart, S., Drummond, M. \& McIntyre, D. (2013). Learning without limits: Constructing a pedagogy free from determinist beliefs about ability. The SAGE handbook of special education, 500515. DOI: 10.4135/9781848607989.n39.

Hong, O. \& Song, J. (2020). A componential model of Science Classroom Creativity (SCC) for understanding collective creativity in the science classroom. Thinking Skills and Creativity, 100698. DOI: 10.1016/j.tsc.2020.100698

Julio, C. (2017). Configurando identidad de aprendiz en la escuela: obstáculos en la relación pedagógica y su incidencia en procesos de "deserción" escolar temprana. Revista Latinoamericana de Educación Inclusiva, 11(1), 109-129. DOI: 10.4067/S071873782017000100008 .

Kaukko, M. \& Wilkinson, J. (2018). "Learning how to go on": refugee students and informal learning practices. International Journal of Inclusive Education, 1-19, 1175-1193. DOI: $10.1080 / 13603116.2018 .1514080$

Kozleski, E., Artiles, A. \& Waitoller, F. (2014). Equity in inclusive education: A cultural historical comparative perspective. In, L. Florian (Ed.), The SAGE handbook of special education (pp. 231-249). New York: Sage.

Ley 20.845. (2015). De inclusión escolar que regula la admisión de los y las estudiantes, elimina el financiamiento compartido y prohíbe el lucro en establecimientos educacionales que reciben aportes del Estado. Biblioteca del Congreso Nacional. Recuperado de: https://www.leychile.cl/Navegar?idNorma=1078 172\&idVersion $=2017-01-28$.

Long, N. (2007). Sociología del desarrollo: una perspectiva centrada en el actor. México D.F.: Ciesas.

López, V., Julio, C., Morales, M., Rojas, C. y Pérez, M. V. (2014). Barreras culturales para la inclusión: políticas y prácticas de integración en Chile. Revista de Educación, 363(1), 256-281. DOI: 10.4438/1988-592X-RE-2012-363-180.

López, V., González, P., Manghi, D., Ascorra, P., Oyanedel, J. C., Redón, S., Leal, F. y Salgado, M. (2018). Políticas de inclusión educativa en Chile: Tres nudos críticos. Education Policy Analysis Archives, 26(157), 1-24. DOI: 10.14507/epaa.26.3088. 
Mertens, D. (2010). Research and evaluation in Education and Psychology. Integrating diversity with quantitative, qualitative and mixed methods. Thousand Oaks: Sage.

Messiou, K. \& Ainscow, M. (2020). Inclusive Inquiry: Student-teacher dialogue as a means of promoting inclusion in schools. British Educational Research Journal, 46(3), 670-687. DOI: 10.1002/berj.3602

Ministerio de Educación. (2010). Guía de apoyo técnico-pedagógico: Necesidades educativas especiales en el nivel de educación parvularia. Santiago, Chile: Gobierno de Chile.

Ministerio de Educación. (2013). Orientaciones Técnicas para programas de Integración Escolar. División de Educación General, Unidad de Educación Especial. Santiago, Chile: Gobierno de Chile.

Monereo, C. (2010). La formación del profesorado: Una pauta para el análisis e intervención a través de incidentes críticos. Revista Iberoamericana de Educación, (52), 149-178. Recuperado de: https://rieoei.org/historico/documentos/rie52a08.pdf.

Monereo, C. y Monte, M. (2011). Docentes en Tránsito: Incidentes críticos en secundaria. Barcelona: Graó.

Muñoz-Abarca, F., Figueroa, I. y Yáñez-Urbina, C. (2020). Escenarios de la Experiencia Escolar: una exploración desde la voz de estudiantes etiquetados con Discapacidad Intelectual. Polyphonia, 4(1), 72-95. Recuperado de: https://revista.celei.cl/ index.php/PREI/article/view/132.

Nail, O., Gajardo, J. y Muñoz, M. (2012). La técnica de análisis de incidentes críticos: Una herramienta para la reflexión sobre prácticas docentes en convivencia escolar. Psicoperspectivas, 11(2), 56-76. DOI: 10.5027/psicoperspectivas-vol11-issue2fulltext-204.

Nyman, J., Parisod, H., Axelin, A. \& Salanterä, S. (2019). Finnish adolescents' self-efficacy in peer interactions: a critical incident study. Health promotion international, 34(5), 961-969. DOI: 10.1093/heapro/day048

Paulín, H., Tomasini, M., D’Aloisio, F., López, C., Nocetti, M. y García, G. (2011). La representación teatral como dispositivo de investigación cualitativa para la indagación de sentidos sobre la experiencia escolar con jóvenes. Psicoperspectivas, 10(2), 134-155. DOI: 10.5027/psicoperspectivas-vol10-issue2-fulltext-149. 
Peña, M. (2013). Análisis crítico del discurso del Decreto 170 de subvención diferenciada para necesidades educativas especiales: El diagnóstico como herramienta de gestión. Psicoperspectivas, 12, 93-103. DOI: 10.2307/j.ctvfc51wz.7.

Prieto M. (2005). La participación de los estudiantes: ¿Un camino hacia su emancipación? Chile. Theoría, 14(1), 27-36. Recuperado de: http://www.ubiobio.cl/theoria/v/v15/a3.pdf.

Ramos, L. (2014). Educación Especial y Educación Inclusiva en Chile: ¿En punto de estancamiento? Revista Latinoamericana de Educación Inclusiva, 7(2), 37-46. Recuperado de: http:// www.rinace.net/rlei/numeros/vol7-num2/art2.pdf.n

Riessman, C.K. (2008). Narrative methods for the human sciences. Los Angeles: Sage.

Salgado, A. (2007). Investigación cualitativa: diseños, evaluación del rigor metodológico y retos. Liberabit, (13), 71-78. Recuperado de: http://www.scielo.org.pe/scielo.php?script=sci_artt ext\&pid=S1729-48272007000100009.

Sevilla, H. \& Gamboa, R. (2017). Critical Incidents, Reflective Writing, and Future Teachers' Professional Identities. Revista de Lenguas Modernas, O(26), 233-256. DOI: 10.15517/rlm.v0i26.29971

Sañudo, M. y Susinos, T. (2018). ¿Quién toma la palabra en la escuela? ¿Quién escucha? Preguntas Pertinentes desde la práctica reflexiva. Revista Latinoamericana de educación inclusiva, 12(1), 79-94. DOI: https://doi.org/10.4067/S071873782018000100079

Susinos, T. y Ceballos, N. (2012). Voz del alumnado y presencia participativa en la vida escolar. Apuntes para una cartografía de la voz del alumnado en la mejora educativa. Revista de Educación, (359), 24-44. DOI: 10.4438/1988-592X-RE-2012359-194.

Susinos, T. y Rodríguez, C. (2011). La educación inclusiva hoy. Reconocer al otro y crear comunidad a través del diálogo y la participación. Revista Interuniversitaria de Formación del profesorado, 25(1). Recuperado de: https://www.redalyc.org/ pdf/274/27419147002.pdf.

Trilla, J. y Novella, A. (2001). Participación, democracia y formación para la ciudadanía. Los consejos de infancia. Revista de Educación, (356), 23-43. Recuperado de: http://www.revistaeducacion.educacion.es/re2011/re2011_02.pdf. 
Tyack, D. y Cuban, L., (2001). En busca de la utopía. Un siglo de reformas de las escuelas públicas. México D.F.: Fondo de Cultura Económica.

Urbina, C., Ipinza Villamán, R. y Gutiérrez-Fuentes, L. (2020). Prácticas relacionales profesor-estudiante y participación en el aula: Desafíos para la construcción de una convivencia democrática. Psicoperspectivas, 19(3), 29-40. DOI: 10.5027/ psicoperspectivas-vol19-issue3-fulltext-2045.

Valdés, M. y Monereo, C. (2012). Desafíos a la formación del docente inclusivo: la identidad profesional y su relación con los incidentes críticos. Revista Latinoamericana de Inclusión Educativa, 6(2), 193-208. Recuperado de: http://www.rinace.net/ rlei/numeros/vol6-num2/art8.pdf.

Vallejos, R. (2013). La técnica de análisis de incidentes críticos: origen y desarrollo. En O. Nail (coord.), Análisis de incidentes críticos de aula. Una herramienta para la mejora de la convivencia (pp. 37-50). Santiago: Ril.

Veiga-Neto, A. (2001). Incluir para saber: Saber para excluir. Pro-Posições-Revista Quadrimestral da Faculdade de Educação, 12(2-3), 22-31. Recuperado de: https://periodicos.sbu.unicamp.br/ojs/index.php/proposic/article/view/8643993.

Vergara, A., Peña, M., Chávez, P. y Vergara, E. (2015). Los niños como sujetos sociales: el aporte de los nuevos estudios sociales de la infancia y el análisis crítico del discurso. Psicoperspectivas, 14(1), 55-65. DOI: 10.5027/PSICOPERSPECTIVAS-VOL14ISSUE1-FULLTEXT-544.

Vetoniemi, J. \& Kärnä, E. (2019). Being included-experiences of social participation of pupils with special education needs in mainstream schools. International Journal of Inclusive Education, 25(10), 1190-1204 https://doi.org/10.1080/13603116.2019.1603329.

Villalobos, B., Carrasco, C., López, V., Bilbao, M. Á., Morales, M., Ortiz, S., Olavarría, D., Ascorra. P. y Ayala del Castillo, Á. (2015). Inclusión y violencia: Prevalencia de victimización entre pares en estudiantes que participan en Programas de Integración Escolar. Revista Latinoamericana de Educación Inclusiva, 8(2), 161-178. Recuperado de: http://www.rinace.net/rlei/ numeros/vol8-num2/art8.pdf.

Willis, C., Girdler, S., Thompson, M., Rosenberg, M., Reid, S. \& Elliott, C. (2017). Elements contributing to meaningful participa- 
tion for children and youth with disabilities: a scoping review. Disability and rehabilitation, 39(17), 1771-1784. DOI: 10.1080/09638288.2016.1207716.

Yañez-Urbina, C., Figueroa, I., Soto, J. y Sciolla, B. (2018). La voz en la mirada: fotovoz como una metodología para explorar los procesos de inclusión-exclusión desde la perspectiva del estudiantado. Pensamiento Educativo, 55(2), 1-16. DOI: 10.7764/pel.55.2.2018.4. 